\title{
Toward Global Quantum Communication: Beam Wandering Preserves Nonclassicality
}

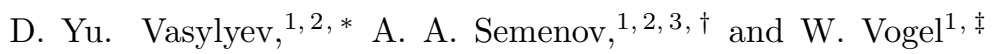 \\ ${ }^{1}$ Institut für Physik, Universität Rostock, Universitätsplatz 3, D-18051 Rostock, Germany \\ ${ }^{2}$ Bogolyubov Institute for Theoretical Physics, NAS of Ukraine, Vul. Metrologichna 14-b, 03680 Kiev, Ukraine \\ ${ }^{3}$ Institute of Physics, NAS of Ukraine, Prospect Nauky 46, 03028 Kiev, Ukraine
}

(Dated: October 15, 2018)

\begin{abstract}
Tap-proof long-distance quantum communication requires a deep understanding of the strong losses in transmission channels. Here we provide a rigorous treatment of the effects of beam wandering, one of the leading disturbances in atmospheric channels, on the quantum properties of light. From first principles we derive the probability distribution of the beam transmissivity, with the aim to completely characterize the quantum state of light. It turns out that beam wandering may preserve nonclassical effects, such as entanglement, quadrature and photon number squeezing, much better than a standard attenuating channel of the same losses.
\end{abstract}

PACS numbers: 42.50.Nn, 42.68.Ay, 42.50.Ar

Introduction.- The quantum properties of light open challenging perspectives for secure data transfer [1]. Quantum communication is already used commercially, but its application is still restricted by relatively small distances. The main limitations are the strong losses over long distances. For example, in optical fibers typical values are $0.2 \mathrm{~dB} / \mathrm{km} \mathrm{[2,} \mathrm{allowing} \mathrm{us} \mathrm{to} \mathrm{preserve} \mathrm{quantum}$ entanglement over $100 \mathrm{~km} \mathrm{3}$. However, even this distance is insufficient for global quantum communication. Some possibilities to improve this situation are based on protocols, which are rather stable against losses [4], or on the idea of quantum repeaters [5].

A promising alternative is based on free-space channels for satellite-based global quantum communication, which can bridge any distance on the Earth. In recent experiments quantum entanglement of light has been demonstrated, after transmission through a free-space channel of $144 \mathrm{~km}$ with atmospheric losses of $32 \mathrm{~dB}$ [6, 7]. Atmospheric losses are instable and permanently fluctuating. The related noise is expected to substantially diminish the quantum properties of the transmitted light. However, such fluctuations may even be useful to preserve quantum entanglement [8].

Noise effects in free-space channels have been extensively studied [9, 10, including models with random fluctuations and stochastic modulation of the intensity $11-$ 14. More recently, the method of fluctuating-loss channels has been introduced [15, based on fluctuations of the complex transmission coefficient, whose absolute value is bounded by one [16, 17]. Beyond the earlier models $11-$ [13, the latter work in Ref. [15] yields a consistent description of quantum channels.

The probability distribution of the transmission coefficient (PDTC) is the main characteristic of fluctuatingloss channels. However, the lack of a consistent model for the PDTC prevents us from exploiting the advantages of

\footnotetext{
* E-mail address: DVasylyev@bitp.kiev.ua

$\dagger$ E-mail address: sem@iop.kiev.ua

$\ddagger$ E-mail address: werner.vogel@uni-rostock.de
}

this theory under realistic conditions. The phenomenological log-normal model was studied for the random intensity modulation [13, but it incorrectly describes the distribution tail with the transmission coefficient being close to 1 . This domain, however, is most important for preserving the quantum effects of light needed for tapproof communication.

For a realistic description of the quantum effects of light in free-space channels, the derivation of a PDTC from first principles is indispensable. Among the unwanted disturbances in free-space channels we will concentrate on the important phenomenon of beam wandering, caused by turbulence [9, 10] and unstable adjustment of the radiation source. We obtain an analytical expression for the transmission coefficient of an aperture. Based on a normal distribution of the beam positioning 14, we derive an analytical form for the corresponding PDTC. Later on, we study the effects of beam wandering on quantum phenomena, such as photon-number and quadrature squeezing, and violations of Bell inequalities. We show that despite of the extra noise, fluctuating loss channels may preserve such nonclassical properties much better than similar constant-loss channels. This result gives a strong argument to favor free-space quantum communication.

Fluctuating-loss channels.- The description of losses in linear quantum optics usually connects annihilation operators of the input and output fields, $\hat{a}_{\text {in }}$ and $\hat{a}_{\text {out }}$, by the standard input-output relation, $\hat{a}_{\text {out }}=T \hat{a}_{\text {in }}+$ $\sqrt{1-T^{2}} \hat{c}$ cf. [18, Appendix A. The operator $\hat{c}$ describes modes of the environment being in the vacuum state. For our further considerations we assume the absence of dephasing. This applies to many experiments, such as photodetection [13 and polarization analysis for testing Bell inequalities [7. One may also perform homodyning, using a local oscillator copropagating with the signal [19, 20]. Hence, the transmission coefficient $T$ is real and positive. Additionally, preserving the commutation relations implies that $T \in[0,1]$. In fluctuating-loss channels, $T$ is a random variable. The corresponding quantum-state input-output relation attains its simplest 
form in the Glauber-Sudarshan $P$ representation 15 (cf. 18, Appendix B),

$$
P_{\text {out }}(\alpha)=\int_{0}^{1} \mathrm{~d} T \mathcal{P}(T) \frac{1}{T^{2}} P_{\text {in }}\left(\frac{\alpha}{T}\right),
$$

where $P_{\text {in }}(\alpha)$ and $P_{\text {out }}(\alpha)$ are the input and output $P$ functions, respectively. Fluctuations of losses are described by the PDTC, $\mathcal{P}(T)$, which we will derive for the scenario of beam wandering.

Aperture transmission coefficient.- For weak absorption, beam-wandering losses are dominant. Typically they are caused by aperture truncation of the light at the receiver. This implies that $T$ in Eq. (1) is the aperture transmission coefficient of a light pulse. We consider the pulse as a superposition of Gaussian beams 21 with different wave numbers $k$, propagating along the $Z$ axis onto the aperture plane at distance $z_{\text {ap }}$ from the source. If beam deflection is caused by imperfect adjustment of the radiation source or by weak turbulence, the beam incidence is normal to the aperture plane to a good approximation. The beam center is deflected by the distance $r$ from the aperture center, see the inset in Fig. 1 .

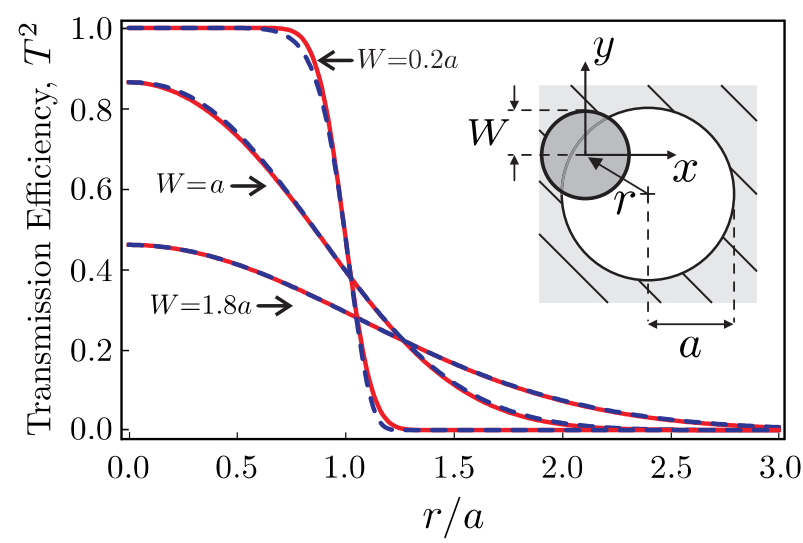

FIG. 1. (color online) Transmission efficiency, $T^{2}$, vs the beam-deflection distance, $r$, for different values of the beamspot radius $W$. Solid and dashed lines correspond to the numerically calculated integral (3) and the analytical approximation (4), respectively. The inset schematically shows the aperture of radius $a$ and the beam-spot of radius $W$.

The transmission efficiency (square of the transmission coefficient) of a Gaussian beam reads as

$$
T^{2}(k)=\int_{\mathcal{A}} \mathrm{d} x \mathrm{~d} y\left|U\left(x, y, z_{\text {ap }} ; k\right)\right|^{2},
$$

where $\mathcal{A}$ is the area of aperture opening, $U\left(x, y, z_{\text {ap }} ; k\right)$ is the Gaussian beam normalized in the $X Y$ plane. In practice the transmission coefficient $T(k)$ approximately equals the pulse transmission coefficient in Eq. (1). That is, the pulse spectrum is irrelevant, $T \approx T\left(k_{0}\right)$, with $k_{0}$ being the carrier wave-number. For a Gaussian beam with the spot radius $W$ at the aperture plane the transmission efficiency is given by the incomplete Weber inte- gral 22],

$$
T^{2}=\frac{2}{\pi W^{2}} e^{-2 \frac{r^{2}}{W^{2}}} \int_{0}^{a} \mathrm{~d} \varrho \varrho e^{-2 \frac{\varrho^{2}}{W^{2}}} \mathrm{I}_{0}\left(\frac{4}{W^{2}} r \varrho\right),
$$

$a$ is the aperture radius and $\mathrm{I}_{n}$ the modified Bessel function. Details can be found in [18, Appendix C.

The integral (3) can be calculated numerically [14. However, for the evaluation of the PDTC we propose an approximate analytical expression of the form (cf. [18, Appendix D)

$$
T^{2}=T_{0}^{2} \exp \left[-\left(\frac{r}{R}\right)^{\lambda}\right]
$$

where $T_{0}$ is the maximal transmission coefficient for the given beam-spot radius $W ; \lambda$ and $R$ are the shape and scale parameter, respectively. The constants $T_{0}, \lambda$, and $R$ are obtained from the following procedure. We consider the transmission efficiency $T^{2}$ as a function $T^{2}(r)$ of the beam-deflection distance $r$. For the integral (3) one can calculate analytically the particular values $T^{2}(0), T^{2}(a)$, and $\left(\mathrm{d} T^{2}(r) / \mathrm{d} r\right)_{r=a}$ cf. [22]. From the condition $T^{2}(0)=$ $T_{0}^{2}$ one gets

$$
T_{0}^{2}=1-\exp \left[-2 \frac{a^{2}}{W^{2}}\right] .
$$

The shape and scale parameters, $\lambda$ and $R$, are obtained from two algebraic equations for the values of $T^{2}$ and its derivative at the point $a$,

$$
\begin{aligned}
\lambda= & 8 \frac{a^{2}}{W^{2}} \frac{\exp \left[-4 \frac{a^{2}}{W^{2}}\right] \mathrm{I}_{1}\left(4 \frac{a^{2}}{W^{2}}\right)}{1-\exp \left[-4 \frac{a^{2}}{W^{2}}\right] \mathrm{I}_{0}\left(4 \frac{a^{2}}{W^{2}}\right)} \\
& \times\left[\ln \left(\frac{2 T_{0}^{2}}{1-\exp \left[-4 \frac{a^{2}}{W^{2}}\right] \mathrm{I}_{0}\left(4 \frac{a^{2}}{W^{2}}\right)}\right)\right]^{-1}, \\
R= & a\left[\ln \left(\frac{2 T_{0}^{2}}{1-\exp \left[-4 \frac{a^{2}}{W^{2}}\right] \mathrm{I}_{0}\left(4 \frac{a^{2}}{W^{2}}\right)}\right)\right]^{-\frac{1}{\lambda}} .
\end{aligned}
$$

In Fig. 1 we compare the numerical result for $T^{2}(r)$ with the approximation (4)-(7). The maximal relative mean quadratic error appears for $W=0.23 a$ and is $1.85 \%$. This gives a reasonable accuracy for the applied technique.

Probability distribution of the transmission coefficient.- We suppose that the beam-center position is normally distributed with variance $\sigma^{2}$ around a point at the distance $d$ from the aperture center. In the case of beam wandering caused by imperfect adjustment of the radiation source this variance is $\sigma^{2} \approx \sigma_{\vartheta}^{2} z^{2}$, where $\sigma_{\vartheta}^{2}$ is the variance of the source-deflection angle. Likewise, in the case of weak atmospheric turbulence, $\sigma^{2} \approx 1.919 C_{n}^{2} z^{3}\left(2 W_{0}\right)^{-1 / 3}\left[10\right.$, where $C_{n}^{2}$ is the indexof-refraction structure constant, $W_{0}$ is the beam-spot radius at the radiation source; for more recent results see [23. Generally, both variances should be added up.

Based on the above assumption, the beam-deflection distance $r$ fluctuates according to the Rice distribution 11 with the parameters $d$ and $\sigma$. Provided that the 
transmission coefficient $T$ is approximated by Eq. (4), the PDTC is given by the log-negative generalized Rice distribution,

$$
\begin{array}{r}
\mathcal{P}(T)=\frac{2 R^{2}}{\sigma^{2} \lambda T}\left(2 \ln \frac{T_{0}}{T}\right)^{\frac{2}{\lambda}-1} \mathrm{I}_{0}\left(\frac{R d}{\sigma^{2}}\left[2 \ln \frac{T_{0}}{T}\right]^{\frac{1}{\lambda}}\right) \\
\times \exp \left[-\frac{1}{2 \sigma^{2}}\left\{R^{2}\left(2 \ln \frac{T_{0}}{T}\right)^{\frac{2}{\lambda}}+d^{2}\right\}\right]
\end{array}
$$

for $T \in\left[0, T_{0}\right]$, and $\mathcal{P}(T)=0$ else. In the particular case when the beam fluctuates around the aperture center, $d=0$, this distribution reduces to the log-negative Weibull distribution,

$$
\mathcal{P}(T)=\frac{2 R^{2}}{\sigma^{2} \lambda T}\left(2 \ln \frac{T_{0}}{T}\right)^{\frac{2}{\lambda}-1} \exp \left[-\frac{1}{2 \sigma^{2}} R^{2}\left(2 \ln \frac{T_{0}}{T}\right)^{\frac{2}{\lambda}}\right],
$$

for $T \in\left[0, T_{0}\right]$, and $\mathcal{P}(T)=0$ else. More details about properties of the PDTC can be found in [18, Appendix E.

The PDTC (8) and (9) may have singularities at the points $T=0$ and $T=T_{0}$. Evidently, in any experimental reconstructions of the $\mathrm{PDTC}$ this singularity cannot be observed directly due to finite values of the samplingdata number and the sampling interval for $T$. For this reason, we will use the exceedance (tail distribution),

$$
\overline{\mathcal{F}}(T)=\int_{T}^{1} \mathrm{~d} T^{\prime} \mathcal{P}\left(T^{\prime}\right),
$$

which is the probability that the transmission coefficient exceeds the value $T$. Plots of this function are presented in Fig. 2. In the following we will show that contributions from tails with large $T$ are important for preserving nonclassical properties of the transmitted light. This implies that the exceedance may also characterize the feasibility of quantum protocols.

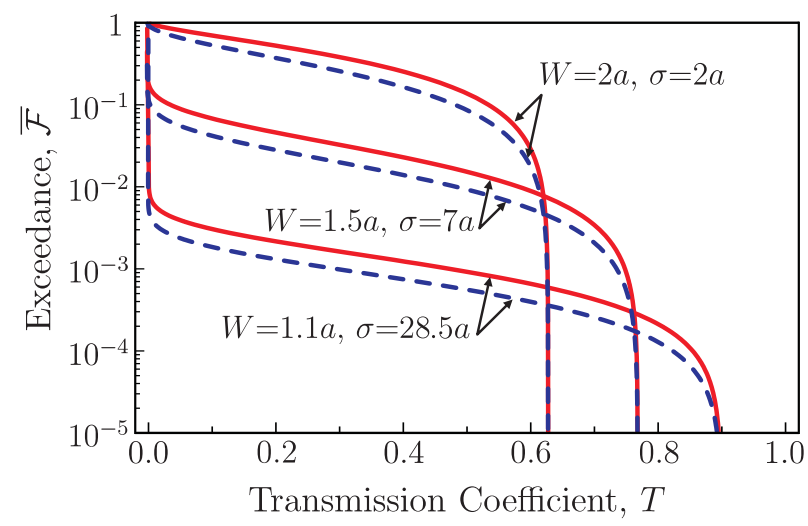

FIG. 2. (color online) The exceedance (tail distribution) cf. Eq. 10 , is shown in the logarithmic scale for different values of the beam-spot radius $W$, and standard deviation of beam deflection $\sigma$. Solid and dashed lines correspond to $d=0$ and $d=\sigma$, respectively.
Nonclassical effects.- Input-output relation (1) and PDTC (8), (9) enable to consistently characterize quantum states of the transmitted light. We will use this possibility for studying disturbances of nonclassical effects by beam wandering. In this context the fluctuating-loss channel with the mean transmission efficiency $\left\langle T^{2}\right\rangle$ is compared with a standard attenuating channel with the transmission efficiency $T^{2}=\left\langle T^{2}\right\rangle$.

A remarkable example is the experimental violation of Bell inequalities, after transmission over a $144 \mathrm{~km}$ atmospheric channel with a mean loss of $32 \mathrm{~dB}$ [7]. As it has been reported in Ref. 8, this result is very sensitive to stray-light and dark counts. For example, such a violation is impossible for constant attenuation of the same size and the mean number of noise counts of $10^{-5}$ (instead of $0.5 \times 10^{-6}$ in Ref. [7]) cf. the dashed line in Fig. 3. Indeed, in this case the required simultaneous clicks of the detectors are mainly caused by stray-light and dark counts. For fluctuating losses events with large $T$, i.e. in the tail of the PDTC, ensure the simultaneous clicks of detectors from an entangled-photon source. A problem is that the earlier models, e.g. the log-normal PDTC, do not give a correct behavior for the tail.

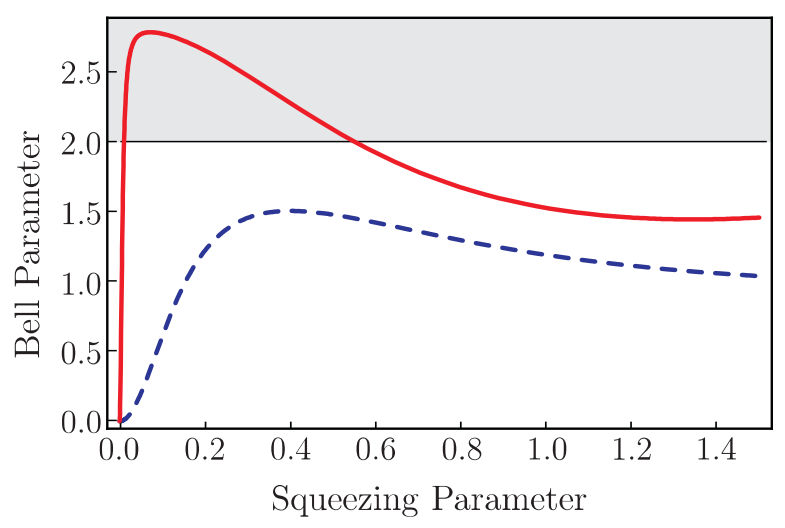

FIG. 3. (color online) The Bell parameter is shown in dependence of the squeezing parameter of a parametric downconversion source. The solid line shows the situation for beam wandering, with a standard deviation $\sigma=28.5 a$ of beam deflection and a beam-spot radius of $W=1.1 a$, leading to a mean loss of $32 \mathrm{~dB}$. The receiver-module losses are supposed to be $9 \mathrm{~dB}$. The dashed line shows the behavior for constant losses of the same size. The mean number of stray-light and dark counts is assumed to be $10^{-5}$. The shaded area indicates the violation of the Bell inequality.

Hence it is important to verify that the tail of a consistent PDTC really improves violations of Bell inequalities, compared with the case of constant losses. In the present consideration we suppose that (on average) $32 \mathrm{~dB}$ of fluctuating losses are caused by beam wandering only. In fact, other phenomena significantly contribute to the measured statistics as well [6. Here we effectively model all of them by the PDTC (9). We also include into consideration $9 \mathrm{~dB}$ losses of the receiver module.

We provide calculations similar to Ref. 8 but for the 
PDTC (9) cf. also 18, Appendix F. The result is given in Fig. 3. It clearly shows that the entanglement can survive in case of beam wandering. Under similar conditions it disappears for constant losses of the same size. This finding is of vital importance for long-distance quantum communication based on entanglement and discrete-variable coding.

The need to observe clicks at two polarization analyzers post-selects also events with large $T$ in the Bellinequality test. In continuous-variable quantum communication based on squeezed states [24, such a natural procedure does not occur. In order to preserve quadrature squeezing we should additionally monitor the transmission coefficient and post-select the events with $T$ exceeding a certain value $T_{\text {min }}$. Likewise, similar procedure can be applied for improving the photon-number squeezing.

Monitoring the transmission coefficient can be performed with the technique proposed in Ref. 20, where a test pulse (also used as a local oscillator [19]) is sent in the mode orthogonally-polarized to the signal. By appropriately choosing $T_{\min }$ we can, in principle, preserve any value of squeezing. However, this is limited by the occurrence of the corresponding events. Hence the feasibility of this procedure must be analyzed.

To be definite, assume that the input light is amplitude-squeezed by $6 \mathrm{~dB}$ and coherently displaced by an amplitude of 10. Similar to Bell inequality test, we suppose that beam wandering leads to $32 \mathrm{~dB}$ mean losses. In standard attenuation channels such losses result in negligibly small squeezing: $8.8 \times 10^{-4} \mathrm{~dB}$ for the quadrature and $8.6 \times 10^{-4} \mathrm{~dB}$ for the photon number. In Fig. 4 we present the value of squeezing in dependence on the exceedance 10 for the point $T_{\min }$, for details of calculations cf. 18, Appendix G. It is clearly seen from the plots that approximately one event from $10^{4}$ is characterized by the high level of squeezing. Hence, under such conditions, the technique is still feasible. This conclusion is of importance for long-distance quantum communication based on continuous-variable coding.

Summary and Conclusions.- Starting from the input-output relations for general quantum states we consistently describe the quantum properties of light undergoing beam wandering in free-space channels. From first principles we have analytically derived the main characteristics of such channels - the probability distribution of the aperture transmission coefficient. It is given by the log-negative generalized Rice distribution. When the centers of beam-wandering and aperture coincide, this function reduces to the log-negative Weibull distribution. The proposed distribution does not include phenomena other than beam wandering, which also may occur in turbulent media. However, our approach is the first model of free-space channels which consistently applies to the quantum domain. We believe that further generalizations will be possible.

It has been shown that fluctuating losses associated with beam wandering can be more favorable for transferring quantum properties of light, compared with con-

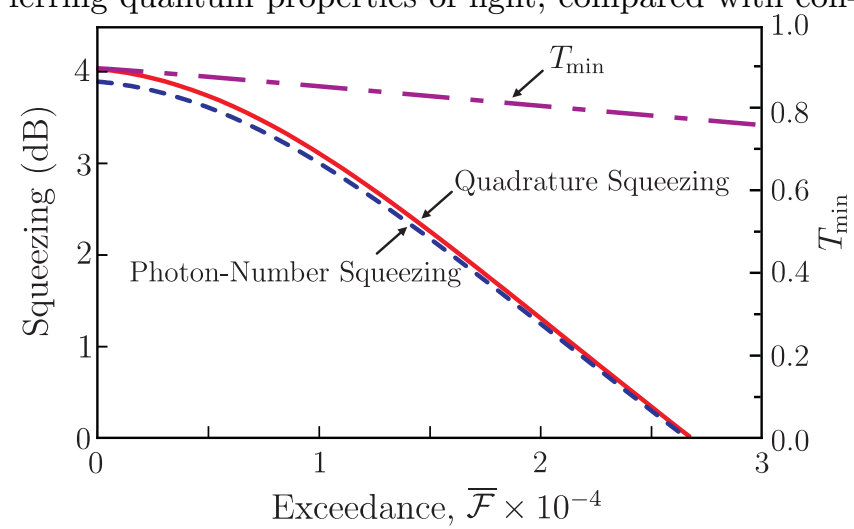

FIG. 4. (color online) The reachable values of quadrature and photon-number squeezing are shown as a function of the corresponding exceedance. The input light is amplitudesqueezed by $6 \mathrm{~dB}$ and coherently displaced by an amplitude of 10 . The mean losses are $32 \mathrm{~dB}$ with the same parameters of beam wandering as in Fig. 3 . The dependence of $T_{\min }$ on the exceedance is also shown.

stant losses of the same size, e.g. in fibers. This includes phenomena such as entanglement, photon-number and quadrature squeezing. Their conservation plays a crucial role for free-space quantum communication based on discrete or continuous variables.

The authors are grateful to Jeffrey H. Shapiro, Alessandro Fedrizzi, and Jan Sperling for useful comments. This work was partly supported by the Deutsche Forschungsgemeinschaft through SFB 652.
[1] N. Gisin and R. Thew, Nature Photon. 1, 165 (2007).

[2] F. Mitschke, Fiber Optics: Physics and Technology (Springer, Berlin, 2009).

[3] H. Hübel et al. Opt. Express 15, 7853 (2007); T. Honjo et al. Opt. Express 15, 13957 (2007); Q. Zhang et al. Opt. Express 16, 5776 (2008).

[4] H. Takesue et al., Nature Photon. 1, 343 (2007).

[5] H.-J. Briegel, W. Dür, J. I. Cirac, and P. Zoller, Phys. Rev. Lett. 81, 5932 (1998); Zhi Zhao, Tao Yang, Yu-Ao Chen, An-Ning Zhang, and Jian-Wei Pan, Phys. Rev.
Lett. 90, 207901 (2003).

6] R. Ursin et al. Nature Phys. 3, 481 (2007).

[7] A. Fedrizzi et al., Nature Phys. 5, 389 (2009).

[8] A. A. Semenov and W. Vogel, Phys. Rev. A 81, 023835 (2010).

[9] V. Tatarskii, The Effect of the Turbulent Atmosphere on Wave Propagation (Israel Program for Scientific Translations, Jerusalem, 1971); A. Ishimaru, Wave Propagation and Scattering in Random Media (Academic Press, NY, 1978). 
[10] R.L. Fante, Proc. IEEE 63, 1669 (1975); 68, 1424 (1980).

[11] E. Jakeman and K. D. Ridley, Modeling Fluctuations in Scattered Waves (Taylor \& Francis Group, NY, 2006).

[12] L. C. Andrews and R. L. Phillips, J. Opt. Soc. Am. A 2, 160 (1985); ibid. 3, 1912 (1986); J. H. Churnside and R. J. Hill, ibid. 4, 727 (1987); J. H. Churnside and S. F. Clifford, ibid. 4, 1923 (1987); J. H. Churnside and R. G. Frehlich, ibid. 6, 1760 (1989); R. J. Hill and R. G. Frehlich, ibid. 14, 1530 (1997); M. A. Al-Habash, L. C. Andrews, and R. L. Phillips, Opt. Eng. 40, 1554 (2001).

[13] P. Diament and M. C. Teich, J. Opt. Soc. Am. 60, 1489 (1970); J. Peřina, Czech. J. Phys. 22, 1075, (1972); J. Peřina, V. Peřinova, M. C. Teich, and P. Diament, Phys. Rev. A 7, 1732 (1973); J. H. Shapiro, B. A. Capron, and R. C. Harney, Appl. Opt. 20 (3292), 1981; J. Peřina, Quantum Statistics of Linear and Nonlinear Optical Phenomena (D. Reidel Publishing Company, 1984); P. W. Milonni, J. H. Carter, Ch. G. Peterson, and R. J. Hughes, J.Opt. B: Quantum Semiclass. Opt. 6, S742 (2004).

[14] R. Esposito, Proc. IEEE 55, 1533 (1967).

[15] A. A. Semenov and W. Vogel, Phys. Rev. A 80, 021802(R) (2009).

[16] R. Dong et al., Nature Physics 4, 919 (2008).

[17] N. D. Hardy and J. H. Shapiro, Phys. Rev. A 84, 063824 (2011).

[18] See Supplemental Material.

[19] D. Elser, T. Bartley, B. Heim, Ch. Wittmann, D. Sych, and G. Leuchs, New J. Phys. 11, 045014 (2009); B. Heim, D. Elser, T. Bartley, M. Sabuncu, Ch. Wittmann, D. Sych, Ch. Marquardt, and G. Leuchs, Appl. Phys. B 98, 635 (2010).

[20] A. A. Semenov, F. Töppel, D. Yu. Vasylyev, H. V. Gomonay, and W. Vogel, Phys. Rev. A 85, 013826 (2012).

[21] L. Mandel and E. Wolf, Optical coherence and quantum optics (Cambridge University Press, Cambridge, 1995).

[22] M. M. Agrest and M. S. Maximov, Theory of Incomplete Cylindrical Functions and their Applications (Springer, Berlin, 1971).

[23] G. P. Berman, A. A. Chumak, and V. N. Gorshkov, Phys. Rev. E. 76, 056606 (2007).

[24] V. Scarani et al., Rev. Mod. Phys. 81, 1301 (2009).

\section{SUPPLEMENTAL MATERIAL}

\section{Appendix A: Operator input-output relation}

The description of linear losses in the absence of dephasing is usually based on the operator input-output relation,

$$
\hat{a}_{\text {out }}=T \hat{a}_{\text {in }}+\sqrt{1-T^{2}} \hat{c},
$$

where $\hat{a}_{\text {in }}$ and $\hat{a}_{\text {out }}$ are the input and output fieldannihilation operators, respectively, $\hat{c}$ is the operator of environment modes, $T \in[0,1]$ is the transmission coefficient. Moreover, $T$ is assumed to be a random variable in the case of fluctuating-loss channels. Expression (A1) can also be considered as the input-output relation in the Heisenberg picture of motion. Here we will show how to derive this relation from the first principles for free-space channels and to get an explicit form for the transmission coefficient.

We suppose that the atmosphere absorption is negligible in the channel. In this case the light is scattered, so that parts of it do not reach the receiver aperture, which leads to losses. The positive-frequency part of the field operators before the aperture (the input field), $\hat{A}_{\text {in }}(\mathbf{r}, t)$, and after the aperture (the output field), $\hat{A}_{\text {out }}(\mathbf{r}, t)$, satisfies the wave equation,

$$
n^{2}(\mathbf{r}) \frac{\partial^{2}}{\partial t^{2}} \hat{A}_{\text {in } / \text { out }}(\mathbf{r}, t)-c^{2} \Delta \hat{A}_{\text {in } / \text { out }}(\mathbf{r}, t)=0,
$$

where $c$ is the speed of light in the vacuum and

$$
n(\mathbf{r})=1+\delta n(\mathbf{r})
$$

is the atmosphere index of refraction. These fields are related to each other as

$$
\hat{A}_{\text {out }}(\mathbf{r}, t)=\int_{-\infty}^{+\infty} \mathrm{d}^{3} \mathbf{r}^{\prime} T\left(\mathbf{r}^{\prime}, \mathbf{r}\right) \hat{A}_{\text {in }}\left(\mathbf{r}^{\prime}, t\right)+\hat{C}(\mathbf{r}, t),
$$

where $T\left(\mathbf{r}^{\prime}, \mathbf{r}\right)$ is the aperture transmission function, $\hat{C}(\mathbf{r}, t)$ is the noise operator associated with the field scattered by the aperture.

Let the input field be initially prepared in the form of a pulse, which corresponds to the normalized nonmonochromatic mode $U_{\text {in }}(\mathbf{r}, t)$. After passing the aperture this pulse transforms to the form

$$
\int_{-\infty}^{+\infty} \mathrm{d}^{3} \mathbf{r}^{\prime} T\left(\mathbf{r}^{\prime}, \mathbf{r}\right) U_{\text {in }}\left(\mathbf{r}^{\prime}, t\right)
$$

The normalization of this pulse gives the corresponding output nonmonochromatic mode,

$$
U_{\text {out }}(\mathbf{r}, t)=\frac{\int_{-\infty}^{+\infty} \mathrm{d}^{3} \mathbf{r}^{\prime} T\left(\mathbf{r}^{\prime}, \mathbf{r}\right) U_{\text {in }}\left(\mathbf{r}^{\prime}, t\right)}{\left(\int_{-\infty}^{+\infty} \mathrm{d}^{3} \mathbf{r}\left|\int_{-\infty}^{+\infty} \mathrm{d}^{3} \mathbf{r}^{\prime} T\left(\mathbf{r}^{\prime}, \mathbf{r}\right) U_{\text {in }}\left(\mathbf{r}^{\prime}, t\right)\right|^{2}\right)^{\frac{1}{2}}},
$$

which is detected and analyzed at the receiver. The functions $U_{\text {in/out }}(\mathbf{r}, t)$ also satisfy the wave equation $\mathrm{A} 2$.

The operators $\hat{A}_{\text {in/out }}(\mathbf{r}, t)$ can be expanded into series of the nonmonochromatic modes,

$$
\begin{aligned}
\hat{A}_{\text {in/out }}(\mathbf{r}, t)=\hat{a}_{\text {in } / \text { out }} & U_{\text {in/out }}(\mathbf{r}, t) \\
& +\sum_{n=1}^{+\infty} \hat{a}_{\text {in/out }}^{(n)} U_{\text {in/out }}^{(n)}(\mathbf{r}, t),
\end{aligned}
$$

where $U_{\text {in/out }}^{(n)}(\mathbf{r}, t)$ form a basis of the orthogonal complement of a mode $U_{\text {in/out }}(\mathbf{r}, t)$, and $\hat{a}_{\text {in/out }}^{(n)}$ are corresponding field-annihilation operators. Let us substitute 
Eq. A7) in Eq. A4). Utilizing the orthogonality condition,

$$
\int_{-\infty}^{+\infty} \mathrm{d}^{3} \mathbf{r} U_{\text {out }}^{(n) *}(\mathbf{r}, t) U_{\text {out }}(\mathbf{r}, t)=0
$$

mode normalization, and Eq. $\mathrm{A} 6$, we get operator input-output relation a1 with the transmission coefficient,

$$
T=\left(\int_{-\infty}^{+\infty} \mathrm{d}^{3} \mathbf{r}\left|\int_{-\infty}^{+\infty} \mathrm{d}^{3} \mathbf{r}^{\prime} T\left(\mathbf{r}^{\prime}, \mathbf{r}\right) U_{\text {in }}\left(\mathbf{r}^{\prime}, t\right)\right|^{2}\right)^{\frac{1}{2}},
$$

being the normalization constant for the output pulse, cf. Eqs. A5 and A6). All terms containing the fieldannihilation operators $\hat{a}_{\text {in }}^{(n)}$ are included in the noise operator $\hat{c}$ of the input-output relation (A1). The corresponding modes also contribute to the output pulse, however, all of them are in the vacuum state.

\section{Appendix B: Quantum-state input-output relations}

In this Appendix we discuss the transformation of the operator input-output relation A1 to the Schrödinger picture of motion, to obtain the corresponding density operators. The easiest way to solve this problem is based on the Glauber-Sudarshan $P$ representation. In this representation the quantum-state input-output relation resembles the corresponding relation in classical optics.

Let us remind that the $P$ function, $P(\alpha)$, is related to the corresponding density operator $\varrho$ as

$$
P(\alpha)=\frac{1}{\pi^{2}} \int_{-\infty}^{+\infty} \mathrm{d}^{2} \beta \Phi(\beta) e^{\alpha \beta^{*}-\alpha^{*} \beta}
$$

where

$$
\Phi(\beta)=\operatorname{Tr}\left[\varrho \exp \left(\hat{a}^{\dagger} \beta\right) \exp \left(-\hat{a} \beta^{*}\right)\right]
$$

is the corresponding characteristic function. By using Eq. (A1), this allows one to derive the relation between the characteristic function of the attenuated and the input field, $\Phi_{T}(\beta)$ and $\Phi_{\text {in }}(\beta)$, respectively, as

$$
\Phi_{T}(\beta)=\Phi_{\text {in }}(T \beta)
$$

Here we utilize the fact that the characteristic function of the environment modes is equal to unity since all these modes are assumed to be in the vacuum state. Substitution of Eq. (B3) in Eq. (B1) gives the input-output relation between the corresponding $P$ functions,

$$
P_{T}(\alpha)=\frac{1}{T^{2}} P_{\text {in }}\left(\frac{\alpha}{T}\right) .
$$

Now we have to take into account the fact that the transmission coefficient $T$ fluctuates randomly. Consequently, we have to average relations $(\mathrm{B} 3)$ and (B4) with the probability distribution of the transmission coefficient (PDTC), $\mathcal{P}(T)$. The resulting input-output relations read as

$$
\begin{gathered}
\Phi_{\text {out }}(\beta)=\int_{0}^{1} \mathrm{~d} T \mathcal{P}(T) \Phi_{\text {in }}(T \beta), \\
P_{\text {out }}(\alpha)=\int_{0}^{1} \mathrm{~d} T \mathcal{P}(T) \frac{1}{T^{2}} P_{\text {in }}\left(\frac{\alpha}{T}\right),
\end{gathered}
$$

where $P_{\text {out }}(\alpha)$ and $\Phi_{\text {out }}(\beta)$ are the $P$ function and the corresponding characteristic function, respectively, of the output field.

The quantum-state input-output relation $\mathrm{B} 6$ can be easily rewritten in any other representations. For example, the corresponding Wigner functions, $W_{\text {in }}(\alpha)$ and $W_{\text {out }}(\alpha)$, are related to each other as

$$
W_{\text {out }}(\alpha)=\int_{0}^{1} \mathrm{~d} T \mathcal{P}(T) \int_{-\infty}^{+\infty} \mathrm{d}^{2} \beta \frac{2}{\pi T^{2}\left(1-T^{2}\right)} \exp \left[-\frac{2|\alpha-\beta|^{2}}{1-T^{2}}\right] W_{\text {in }}\left(\frac{\beta}{T}\right)
$$

which requires an additional $\beta$ integration. It is also useful to have the corresponding relation for the normalordered moments, $M_{n, m}=\operatorname{Tr}\left(\varrho \hat{a} \hat{a}^{\dagger n} \hat{a}^{m}\right)$,

$$
M_{n, m}^{\text {out }}=\left\langle T^{n+m}\right\rangle M_{n, m}^{\text {in }},
$$

which directly leads to Eqs. G6 and G7 of Appendix G.

\section{Appendix C: Pulsed Gaussian beam}

In this Appendix we specify the form of the transmission coefficient A9 for the case of the input pulse prepared as a superposition of Gaussian beams, see Ref. [21, 
with different wave numbers $k$,

$$
\begin{aligned}
& U_{\mathrm{in}}(\mathbf{r}, t) \\
& \quad=\frac{1}{\sqrt{2 \pi}} \int_{-\infty}^{+\infty} \mathrm{d} k C(k) S_{\mathrm{in}}(x, y, z ; k) \exp [i k(z-c t)]
\end{aligned}
$$

where $C(k)$ is the pulse spectrum, $S_{\text {in }}(x, y, z ; k)$ is the input-beam envelope. The beams propagation direction corresponds to the $Z$-axis. Many laser-based sources emit light, which can be approximately described by Eq. C1). The non-normalized pulse after the aperture, cf. Eq. (A5), has the form similar to Eq. (C1),

$$
\begin{aligned}
& \int_{-\infty}^{+\infty} \mathrm{d}^{3} \mathbf{r}^{\prime} T\left(\mathbf{r}^{\prime}, \mathbf{r}\right) U_{\text {in }}\left(\mathbf{r}^{\prime}, t\right) \\
& =\frac{1}{\sqrt{2 \pi}} \int_{-\infty}^{+\infty} \mathrm{d} k C(k) S_{\text {out }}(x, y, z ; k) \exp [i k(z-c t)]
\end{aligned}
$$

where $S_{\text {out }}(x, y, z ; k)$ is the output-beam envelope.

Since the pulses (C1) and (C2) obey the wave equation A2, the input- and output-beam envelopes in the paraxial approximation satisfy the equation

$$
\left[i \frac{\partial}{\partial z}+\frac{1}{2 k} \Delta_{\perp}+k \delta n(\mathbf{r})\right] S_{\text {in } / \text { out }}(x, y, z ; k)=0,
$$

for details see Ref. 21. Here $\Delta_{\perp}$ is the transverse part of the Laplace operator. This equation preserves the norm in the $(X, Y)$ plane, i.e. the relation $\int_{-\infty}^{+\infty} \mathrm{d} x \mathrm{~d} y\left|S_{\text {in/out }}(x, y, z ; k)\right|^{2}$ does not depend on $z$ and equals to unity for the input pulse. The statement that the source irradiates Gaussian beams means that $S_{\text {in }}(x, y, z ; k)$ is the solution of Eq. C3), with the following condition at $z=0$ :

$$
\begin{aligned}
& S_{\mathrm{in}}(x, y, 0 ; k) \\
& \quad=\sqrt{\frac{2}{\pi W_{0}^{2}}} \exp \left[-\left(\frac{1}{2 W_{0}^{2}}+\frac{i k}{2 R_{0}}\right)\left(x^{2}+y^{2}\right)\right] e^{i \Psi_{0}},
\end{aligned}
$$

where $W_{0}, R_{0}$, and $\Psi_{0}$ are values at the transmitter for the beam-spot radius, curvature radius of the wavefront, and the Gouy phase, respectively.

We substitute Eq. C2 in Eq. A9 and take into account the fact that $S_{\text {out }}(x, y, z ; k)$ is slowly varying with respect to $z$, comparing with $\exp [i k z]$. Hence, in the corresponding integration we can approximate $S_{\text {out }}(x, y, z ; k)$ by $S_{\text {out }}\left(x, y, z_{\text {ap }} ; k\right)$, where $z_{\text {ap }}$ is the position of the aperture plane at the $Z$-axis. This results in the fact that the transmission efficiency (square of the transmission coefficient) has the form of decomposition,

$$
T^{2}=\int_{-\infty}^{+\infty} \mathrm{d} k|C(k)|^{2} T^{2}(k)
$$

of the beam transmission efficiencies,

$$
T^{2}(k)=\int_{-\infty}^{+\infty} \mathrm{d} x \mathrm{~d} y\left|S_{\text {out }}\left(x, y, z_{\mathrm{ap}} ; k\right)\right|^{2},
$$

with different wave numbers $k$.

At the aperture plane, $z=z_{\text {ap }}$, the input and output beam envelops are related to each other as

$$
S_{\text {out }}\left(x, y, z_{\text {ap }} ; k\right)=\left\{\begin{array}{ll}
S_{\text {in }}\left(x, y, z_{\text {ap }} ; k\right), & \text { for }(x, y) \in \mathcal{A} \\
0, & \text { for }(x, y) \notin \mathcal{A}
\end{array},\right.
$$

where $\mathcal{A}$ is the area of aperture opening. This implies that Eq. C6) reduces to

$$
T^{2}(k)=\int_{\mathcal{A}} \mathrm{d} x \mathrm{~d} y\left|S_{\text {in }}\left(x, y, z_{\text {ap }} ; k\right)\right|^{2} .
$$

Here the integration is taken over the area of aperture opening.

In practice, the shape of the pulse spectrum, $|C(k)|^{2}$, is much narrower than the shape of the beam transmission efficiency, $T^{2}(k)$. Besides, as it follows from the normalization condition for the input pulse,

$$
\int_{-\infty}^{+\infty} \mathrm{d} k|C(k)|^{2}=1
$$

This yields that the pulse transmission efficiency C5 is approximately equal to the beam transmission efficiency (C8), $T^{2} \approx T^{2}\left(k_{0}\right)$, where $k_{0}$ is the carrier wavenumber. Remaining that the Gaussian-beam amplitude is

$$
U(x, y, z ; k)=S_{\text {in }}(x, y, z ; k) \exp (i k z),
$$

the transmission efficiency can be equivalently given by

$$
T^{2} \approx T^{2}\left(k_{0}\right)=\int_{\mathcal{A}} \mathrm{d} x \mathrm{~d} y\left|U\left(x, y, z_{\text {ap }} ; k_{0}\right)\right|^{2},
$$

cf. Eq. (2).

Therefore, in order to find the transmission coefficient one has to resolve Eq. (C3) with the boundary condition (C4). For $\delta n(\mathbf{r})=0$ the solution is obtained by replacing the parameters $W_{0}, R_{0}$, and $\Psi_{0}$ in the boundary condition (C4) with the corresponding values $W, R$, and $\Psi$, which depend on $z$, see Ref. 21] for details. We model the effect of $\delta n(\mathbf{r})$ in Eq. C3 by deflection of the beam from the aperture center, which works for the week turbulence only. Moreover, the beam can also be deflected due to the imperfect source adjustment. Since the aperture form is circular, all directions appear to be equivalent. Hence, we can consider, without loss of generality, the beam-deflection by the distance $r$ in the direction of $X$-axis. The obtained solution,

$$
\begin{aligned}
& S_{\text {in }}(x, y, z ; k) \\
& \quad=\sqrt{\frac{2}{\pi W^{2}}} \exp \left\{-\left[\frac{1}{2 W^{2}}+\frac{i k}{2 R}\right]\left[(x-r)^{2}+y^{2}\right]\right\} e^{i \Psi},
\end{aligned}
$$


should be substituted in Eqs. C10 and C11. This results in the transmission coefficient expressed in terms of the incomplete Weber integral, which is approximately evaluated in Appendix D

$$
T^{2}=\frac{2}{\pi W^{2}} e^{-2 \frac{r^{2}}{W^{2}}} \int_{0}^{a} d \rho \rho e^{-2 \frac{\rho^{2}}{W^{2}}} \mathrm{I}_{0}\left(4 \frac{r \rho}{W^{2}}\right),
$$

where $a$ is the aperture radius, and $\mathrm{I}_{0}$ is the modified Bessel function, cf. Eq. (3).

\section{Appendix D: Incomplete Weber integral}

In this Appendix we give some technical details related to the approximate analytical evaluation of the integral in Eq. C13), see also Eq. (3). First note that the incomplete Weber integral is defined as, see Ref. [22,

$$
\widetilde{Q}_{n}(x, z)=(2 x)^{-n-1} e^{x} \int_{0}^{z} d t t^{n+1} \exp \left(-\frac{t^{2}}{4 x}\right) \mathrm{I}_{n}(t),
$$

where $\mathrm{I}_{n}$ is the modified Bessel function. In terms of this special function Eq. C13 can also be given by

$$
T^{2}=e^{-4 \frac{r^{2}}{W^{2}}} \widetilde{Q}_{0}\left(2 \frac{r^{2}}{W^{2}}, 4 \frac{r a}{W^{2}}\right) .
$$

An alternative representation for $\widetilde{Q}_{n}(x, z)$ consists in the Lommel series of the modified Bessel functions. However, this series converges slowly and for practical purposes one usually uses the numerical integration.

We derive an analytical approximation for Eqs. C13 and (D2). For this purpose we consider the transmission efficiency, $T^{2}$, as a function $T^{2}(r)$ of the variable $r$. This function can be effectively approximated as

$$
T^{2}(r)=T_{0}^{2} \exp \left[-\left(\frac{r}{R}\right)^{\lambda}\right]
$$

cf. Eq. (4), where the parameters $T_{0}, R$, and $\lambda$ (maximal transmission coefficient, scale, and shape parameters, respectively) can be obtained with using some properties of the incomplete Weber integral.

The maximal transmission coefficient $T_{0}$ is obtained by comparing the exact, cf. Eq. (C13), and approximate, cf. Eq. (D3), expressions for $T^{2}(r)$ at the point $r=0$. In this case the integral $\mathrm{C} 13$ is evaluated analytically. Using the straightforward calculations, the maximal transmission efficiency,

$$
T_{0}^{2}=1-\exp \left[-2 \frac{a^{2}}{W^{2}}\right],
$$

cf. Eq. (5), is obtained explicitly.

Similar consideration can be applied for scale, $R$, and shape, $\lambda$, parameters. In this case we compare exact and approximate expressions for $T^{2}(r)$ and their derivatives $\mathrm{d} T^{2}(r) / \mathrm{d} r$ at the point $r=a$. The corresponding integrals for the exact relation (D2) are evaluated explicitly as, cf. Ref. 22,

$$
T^{2}(a)=\frac{1}{2}\left\{1-e^{-4 \frac{a^{2}}{W^{2}}} \mathrm{I}_{0}\left(4 \frac{a^{2}}{W^{2}}\right)\right\},
$$

$$
\begin{aligned}
\left(\frac{\mathrm{d} T^{2}(r)}{\mathrm{d} r}\right)_{r=a}= & \frac{4 a}{\pi W^{2}} e^{-4 \frac{a^{2}}{W^{2}}}\left\{\widetilde{Q}_{1}\left(\frac{2 a^{2}}{W^{2}}, \frac{4 a^{2}}{W^{2}}\right)\right. \\
& \left.-\widetilde{Q}_{0}\left(\frac{2 a^{2}}{W^{2}}, \frac{4 a^{2}}{W^{2}}\right)\right\} \\
= & -\frac{4 a}{w^{2}} e^{-4 \frac{a^{2}}{W^{2}}} \mathrm{I}_{1}\left(4 \frac{r a}{W^{2}}\right) .
\end{aligned}
$$

These expressions can be equated with the same quantities evaluated from the approximate form (D3),

$$
T^{2}(a)=T_{0}^{2} \exp \left[-\left(\frac{a}{R}\right)^{\lambda}\right]
$$

$$
\left(\frac{\mathrm{d} T^{2}(r)}{\mathrm{d} r}\right)_{r=a}=-T_{0}^{2} \frac{\lambda}{R}\left(\frac{a}{R}\right)^{\lambda-1} \exp \left[-\left(\frac{a}{R}\right)^{\lambda}\right] .
$$

The obtained system of two algebraic equations for $R$ and $\lambda$ yields

$$
\begin{aligned}
\lambda= & 8 \frac{a^{2}}{W^{2}} \frac{\exp \left[-4 \frac{a^{2}}{W^{2}}\right] \mathrm{I}_{1}\left(4 \frac{a^{2}}{W^{2}}\right)}{1-\exp \left[-4 \frac{a^{2}}{W^{2}}\right] \mathrm{I}_{0}\left(4 \frac{a^{2}}{W^{2}}\right)} \\
& \times\left[\ln \left(\frac{2 T_{0}^{2}}{1-\exp \left[-4 \frac{a^{2}}{W^{2}}\right] \mathrm{I}_{0}\left(4 \frac{a^{2}}{W^{2}}\right)}\right)\right]^{-1}, \\
R= & a\left[\ln \left(\frac{2 T_{0}^{2}}{1-\exp \left[-4 \frac{a^{2}}{W^{2}}\right] \mathrm{I}_{0}\left(4 \frac{a^{2}}{W^{2}}\right)}\right)\right]^{-\frac{1}{\lambda}},
\end{aligned}
$$

cf. Eqs. (7) and (8).

\section{Appendix E: Beam-wandering PDTC}

In this Appendix we present some technical details related to deriving the beam-wandering PDTC, cf. Eqs. (8) and (9), and discuss mathematical techniques for operating with this object. The basic assumption is that the beam-center position, $\left(x_{o}, y_{o}\right)$, randomly fluctuates according to the two-dimensional Gaussian distribution,

$$
p\left(x_{o}, y_{o} ; \mathbf{d}, \sigma\right)=\frac{1}{2 \pi \sigma^{2}} \exp \left[-\frac{\left(x_{o}-d_{x}\right)^{2}+\left(y_{o}-d_{y}\right)^{2}}{2 \sigma^{2}}\right],
$$

where $\sigma$ is the standard deviation of beam deflection and $\mathbf{d}=\left(d_{x}, d_{y}\right)$ is the mean position of beam center. This 
implies that the beam-deflection distance $r=\sqrt{x_{o}^{2}+y_{o}^{2}}$ fluctuates according to the Rice distribution,

$$
p(r ; d, \sigma)=\frac{r}{\sigma^{2}} \mathrm{I}_{0}\left(\frac{r d}{\sigma^{2}}\right) \exp \left[-\frac{r^{2}+d^{2}}{2 \sigma^{2}}\right],
$$

where $d=\sqrt{d_{x}^{2}+d_{y}^{2}}$ is the distance between aperture and fluctuation centers, $\mathrm{I}_{0}$ is the modified Bessel function.

The random variables $r$ and $T$ are related to each other via Eq. (D3), cf. also Eq. (4). This yields that the probability distribution of the transmission coefficient (PDTC) reads as

$$
\begin{array}{r}
\mathcal{P}(T)=\frac{2 R^{2}}{\sigma^{2} \lambda T}\left(2 \ln \frac{T_{0}}{T}\right)^{\frac{2}{\lambda}-1} \mathrm{I}_{0}\left(\frac{R d}{\sigma^{2}}\left[2 \ln \frac{T_{0}}{T}\right]^{\frac{1}{\lambda}}\right) \\
\times \exp \left[-\frac{1}{2 \sigma^{2}}\left\{R^{2}\left(2 \ln \frac{T_{0}}{T}\right)^{\frac{2}{\lambda}}+d^{2}\right\}\right],
\end{array}
$$

for $T \in\left[0, T_{0}\right]$, and $\mathcal{P}(T)=0$ else, cf. Eq. (8). For this distribution we refer to as the log-negative generalized Rice distribution. This is also important to note that $\log$-losses $\theta=-\ln T^{2}$ are distributed according to the generalized Rice distribution,

$$
\begin{aligned}
\mathcal{P}(\theta)= & \frac{R^{2}}{\sigma^{2} \lambda}\left(\theta-\theta_{0}\right)^{\frac{2}{\lambda}-1} \mathrm{I}_{0}\left(\frac{R d}{\sigma^{2}}\left(\theta-\theta_{0}\right)^{\frac{1}{\lambda}}\right) \\
& \times \exp \left[-\frac{1}{2 \sigma^{2}}\left(R^{2}\left(\theta-\theta_{0}\right)^{\frac{2}{\lambda}}+d^{2}\right)\right],
\end{aligned}
$$

for $\theta \geq \theta_{0}$ and $\mathcal{P}(\theta)=0$ else, where $\theta_{0}=-\ln T_{0}^{2}$. A particular case of Eqs. (8) and (E4) for $d=0$ is the log-negative Weibull distribution for the transmission coefficient,

$$
\mathcal{P}(T)=\frac{2 R^{2}}{\sigma^{2} \lambda T}\left(2 \ln \frac{T_{0}}{T}\right)^{\frac{2}{\lambda}-1} \exp \left[-\frac{1}{2 \sigma^{2}} R^{2}\left(2 \ln \frac{T_{0}}{T}\right)^{\frac{2}{\lambda}}\right]
$$

for $T \in\left[0, T_{0}\right]$, and $\mathcal{P}(T)=0$ else, cf. Eq. (9), and the Weibull distribution with the shape parameter $(\sqrt{2} \sigma / R)^{\lambda}$ and the scale parameter $2 / \lambda$ for log-losses,

$$
\mathcal{P}(\theta)=\frac{R^{2}}{\sigma^{2} \lambda}\left(\theta-\theta_{0}\right)^{\frac{2}{\lambda}-1} \exp \left[-\frac{1}{2 \sigma^{2}} R^{2}\left(\theta-\theta_{0}\right)^{\frac{2}{\lambda}}\right]
$$

for $\theta \geq \theta_{0}$ and $\mathcal{P}(\theta)=0$ else.

Integrations over the PDTC $(8)$ and $(9)$ is convenient to perform with respect to the measure $\mathrm{d} r(T)$. Here

$$
r(T)=R\left(2 \ln \frac{T_{0}}{T}\right)^{\frac{1}{\lambda}}
$$

inverts Eq. (D3). In practice this means that the mean value for the function $f(T)$ of the transmission coefficient $T$ can be obtained as

$$
\int_{0}^{1} \mathrm{~d} T \mathcal{P}(T) f[T]=\int_{0}^{+\infty} \mathrm{d} r p(r ; d, \sigma) f[T(r)],
$$

where $p(r ; d, \sigma)$ is the Rice distribution.
Fluctuations of the transmission coefficient can also be characterized by the cumulative probability distribution,

$$
\mathcal{F}(T)=\int_{0}^{T} \mathrm{~d} T^{\prime} \mathcal{P}\left(T^{\prime}\right)
$$

For our purposes, however, it is convenient to use the exceedance (tail distribution), which is defined as

$$
\overline{\mathcal{F}}(T)=1-\mathcal{F}(T)=\int_{T}^{1} \mathrm{~d} T^{\prime} \mathcal{P}\left(T^{\prime}\right)
$$

cf. Eq. (10). Clearly, the exceedance is equal to the probability that the transmission coefficient exceeds the value of $T$.

The exceedance of log-negative Rice distribution (8) is expressed in terms of the incomplete Weber integral (D1),

$$
\overline{\mathcal{F}}(T)=1-\exp \left[-\frac{d^{2}}{\sigma^{2}}\right] \widetilde{Q}_{0}\left(\frac{d^{2}}{2 \sigma^{2}}, \frac{r(T) d}{\sigma^{2}}\right),
$$

where $r(T)$ is given by Eq. (E7). For this special function we can apply the technique presented in Appendix D. This results in an approximate expression,

$$
\overline{\mathcal{F}}(T)=1-\mathcal{F}_{0}(T) \exp \left[-\left(\frac{d}{D(T)}\right)^{\mu(T)}\right],
$$

where

$$
\mathcal{F}_{0}(T)=1-\exp \left[-\frac{r^{2}(T)}{2 \sigma^{2}}\right]
$$

$$
\begin{gathered}
D(T)=r(T)\left\{\ln \left[\frac{2 \mathcal{F}_{0}(T)}{1-\mathrm{I}_{0}\left(\frac{r^{2}(T)}{\sigma^{2}}\right) \exp \left[-\frac{r^{2}(T)}{\sigma^{2}}\right]}\right]\right\}_{(\mathrm{E} 14}^{-\frac{1}{\mu(T)}}, \\
\mu(T)=\frac{2 r^{2}(T)}{\sigma^{2}} \frac{\mathrm{I}_{1}\left(\frac{r^{2}(T)}{\sigma^{2}}\right)}{1-\mathrm{I}_{0}\left(\frac{r^{2}(T)}{\sigma^{2}}\right) \exp \left[-\frac{r^{2}(T)}{\sigma^{2}}\right]} e^{-\frac{r^{2}(T)}{\sigma^{2}}} \\
\times\left\{\ln \left[\frac{2 \mathcal{F}_{0}(T)}{1-\mathrm{I}_{0}\left(\frac{r^{2}(T)}{\sigma^{2}}\right) \exp \left[-\frac{r^{2}(T)}{\sigma^{2}}\right]}\right]\right\}^{-1} .
\end{gathered}
$$

For $d=0$ the approximate expression $(\mathrm{E} 12)$ reduces to

$$
\overline{\mathcal{F}}(T)=1-\mathcal{F}_{0}(T)=\exp \left[-\frac{r^{2}(T)}{2 \sigma^{2}}\right],
$$

which also coincides with the exact form of the exceedance for the log-negative Weibull distribution (9). 


\section{Appendix F: Bell-inequality test}

In this Appendix we remind technical details from Ref. 8] concerning calculations of the Bell parameter for the light generated by a parametric down-conversion source, transmitted through the atmosphere, and then treated by polarization analyzers with on/off detectors. We consider experimental configuration used in Ref. [7, cf. also Fig. F1. In this scenario both receivers are situated at the same place and photons are split by a small time interval.

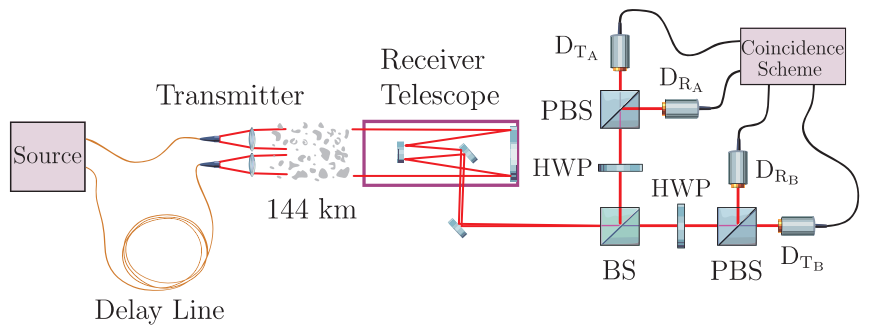

FIG. F1. Setup for checking Bell inequalities with the light transmitted through a $144 \mathrm{~km}$ atmospheric channel, cf. Ref. 7]. A parametric down-conversion source produces entangled photon pairs. These photons, separated by a small time interval, are sent to the receiver. After collecting by the telescope, photons are split by a beam-splitter BS and then treated by the corresponding polarization analyzers. Each polarization analyzer consists of (i) half-wave plate, HWP, which changes the polarization angles $\theta_{\mathrm{A}}$ and $\theta_{\mathrm{B}}$; (ii) polarizing beam-splitter, PBS; (iii) on/off detectors $\mathrm{D}_{\mathrm{T}_{\mathrm{A}}}, \mathrm{D}_{\mathrm{T}_{\mathrm{B}}}$ for the transmitted light, and $\mathrm{D}_{\mathrm{R}_{\mathrm{A}}}, \mathrm{D}_{\mathrm{R}_{\mathrm{B}}}$ for the reflected light.

The Bell inequality in the Clauser-Horne-ShimonyHolt $(\mathrm{CHSH})$ form states that the parameter

$$
\begin{aligned}
\mathcal{B} & =\left|E\left(\theta_{\mathrm{A}}^{(1)}, \theta_{\mathrm{B}}^{(1)}\right)-E\left(\theta_{\mathrm{A}}^{(1)}, \theta_{\mathrm{B}}^{(2)}\right)\right| \\
& +\left|E\left(\theta_{\mathrm{A}}^{(2)}, \theta_{\mathrm{B}}^{(2)}\right)+E\left(\theta_{\mathrm{A}}^{(2)}, \theta_{\mathrm{B}}^{(1)}\right)\right|,
\end{aligned}
$$

also referred to as the Bell parameter, cannot exceed the value of 2 in any local realistic theory. Quantum theory violates the Bell inequality. The correlation coefficients $E\left(\theta_{\mathrm{A}}, \theta_{\mathrm{B}}\right)$ in Eq. (F1) are defined as

$$
E\left(\theta_{\mathrm{A}}, \theta_{\mathrm{B}}\right)=\frac{P_{\text {same }}\left(\theta_{\mathrm{A}}, \theta_{\mathrm{B}}\right)-P_{\text {different }}\left(\theta_{\mathrm{A}}, \theta_{\mathrm{B}}\right)}{P_{\text {same }}\left(\theta_{\mathrm{A}}, \theta_{\mathrm{B}}\right)+P_{\text {different }}\left(\theta_{\mathrm{A}}, \theta_{\mathrm{B}}\right)},
$$

where

$$
P_{\text {same }}\left(\theta_{\mathrm{A}}, \theta_{\mathrm{B}}\right)=P_{\mathrm{T}_{\mathrm{A}}, \mathrm{T}_{\mathrm{B}}}\left(\theta_{\mathrm{A}}, \theta_{\mathrm{B}}\right)+P_{\mathrm{R}_{\mathrm{A}}, \mathrm{R}_{\mathrm{B}}}\left(\theta_{\mathrm{A}}, \theta_{\mathrm{B}}\right)
$$

is the probability to get clicks on detectors in the same channels of the polarization analyzers, and

$$
P_{\text {different }}\left(\theta_{\mathrm{A}}, \theta_{\mathrm{B}}\right)=P_{\mathrm{T}_{\mathrm{A}}, \mathrm{R}_{\mathrm{B}}}\left(\theta_{\mathrm{A}}, \theta_{\mathrm{B}}\right)+P_{\mathrm{R}_{\mathrm{A}}, \mathrm{T}_{\mathrm{B}}}\left(\theta_{\mathrm{A}}, \theta_{\mathrm{B}}\right)
$$

is the probability to get clicks on detectors in different channels. Here $P_{\mathrm{i}_{\mathrm{A}}, \mathrm{i}_{\mathrm{B}}}\left(\theta_{\mathrm{A}}, \theta_{\mathrm{B}}\right)$ is the probability of registering clicks at the detectors $i_{\mathrm{A}}=\left\{\mathrm{T}_{\mathrm{A}}, \mathrm{R}_{\mathrm{A}}\right\}$ and
$i_{\mathrm{B}}=\left\{\mathrm{T}_{\mathrm{B}}, \mathrm{R}_{\mathrm{B}}\right\}$ for the polarization angles $\theta_{\mathrm{A}}$ and $\theta_{\mathrm{B}}$. According to the photodetection theory, these probabilities are given by

$$
P_{i_{\mathrm{A}}, i_{\mathrm{B}}}\left(\theta_{\mathrm{A}}, \theta_{\mathrm{B}}\right)=\sum_{n, m=1}^{+\infty} \operatorname{Tr}\left(\hat{\Pi}_{i_{\mathrm{A}}}^{(n)} \hat{\Pi}_{i_{\mathrm{B}}}^{(m)} \hat{\Pi}_{j_{\mathrm{A}}}^{(0)} \hat{\Pi}_{j_{\mathrm{B}}}^{(0)} \hat{\varrho}\right)
$$

$i_{\mathrm{A}} \neq j_{\mathrm{A}}, i_{\mathrm{B}} \neq j_{\mathrm{B}}$, where $\hat{\varrho}$ is the density operator,

$$
\hat{\Pi}_{i_{\mathrm{A}(\mathrm{B})}^{(n)}}=: \frac{\left(\eta \hat{n}_{i_{\mathrm{A}(\mathrm{B})}}+N\right)^{n}}{n !} \exp \left[-\eta \hat{n}_{i_{\mathrm{A}(\mathrm{B})}}-N\right]:
$$

is the positive operator-valued measure for the detector $i_{\mathrm{A}(\mathrm{B})}, N$ is the mean number of stray-light and dark counts [Semenov et al., Phys. Rev. A 78, 055803 (2008)], $\eta$ is the detection efficiency, : $\cdots$ : means normal ordering, and

$$
\hat{n}_{i_{\mathrm{A}(\mathrm{B})}}=\hat{a}_{i_{\mathrm{A}(\mathrm{B})}^{\dagger}}^{\dagger} \hat{a}_{i_{\mathrm{A}(\mathrm{B})}} .
$$

are the photon-number operators at the outputs of the polarizing beam-splitters PBS. The operator inputoutput relations of the polarization analyzers,

$$
\begin{gathered}
\hat{a}_{T_{\mathrm{A}(\mathrm{B})}}=\hat{a}_{\mathrm{H}_{\mathrm{A}(\mathrm{B})}} \cos \theta_{\mathrm{A}(\mathrm{B})}+\hat{a}_{\mathrm{V}_{\mathrm{A}(\mathrm{B})}} \sin \theta_{\mathrm{A}(\mathrm{B})}, \\
\hat{a}_{R_{\mathrm{A}(\mathrm{B})}}=-\hat{a}_{\mathrm{H}_{\mathrm{A}(\mathrm{B})}} \sin \theta_{\mathrm{A}(\mathrm{B})}+\hat{a}_{\mathrm{V}_{\mathrm{A}(\mathrm{B})}} \cos \theta_{\mathrm{A}(\mathrm{B})},
\end{gathered}
$$

relate the operators $\hat{a}_{i_{\mathrm{A}(\mathrm{B})}}$ with the field operators of the corresponding horizontal and vertical modes on the inputs of the polarization analyzers, $\hat{a}_{\mathrm{H}_{\mathrm{A}(\mathrm{B})}}$ and $\hat{a}_{\mathrm{v}_{\mathrm{A}(\mathrm{B})}}$, respectively. Finally, we utilize the input-output relation for the atmospheric channel, cf. Eq. (A1), and express the operators $\hat{a}_{\mathrm{H}_{\mathrm{A}(\mathrm{B})}}$ and $\hat{a}_{\mathrm{V}_{\mathrm{A}(\mathrm{B})}}$ via the field-operators $\hat{a}_{\mathrm{H}_{\mathrm{A}(\mathrm{B})}}^{\text {in }}$ and $\hat{a}_{\mathrm{v}_{\mathrm{A}(\mathrm{B})}}^{\text {in }}$ at the transmitter,

$$
\begin{aligned}
& \hat{a}_{\mathrm{H}_{\mathrm{A}(\mathrm{B})}}=T \hat{a}_{\mathrm{H}_{\mathrm{A}(\mathrm{B})}}^{\mathrm{in}}+\sqrt{1-T^{2}} \hat{c}_{\mathrm{H}_{\mathrm{A}(\mathrm{B})}}, \\
& \hat{a}_{\mathrm{V}_{\mathrm{A}(\mathrm{B})}}=T \hat{a}_{\mathrm{v}_{\mathrm{A}(\mathrm{B})}}^{\mathrm{in}}+\sqrt{1-T^{2}} \hat{c}_{\mathrm{V}_{\mathrm{A}(\mathrm{B})}},
\end{aligned}
$$

where the fluctuating transmission coefficient $T$ is equal for all four modes due to negligible depolarization in the Earth atmosphere and small time delay between entangled photons.

A special part of the device is the 50:50 beam-splitter BS, cf. Fig. F1, which randomly separates entangled photons. The devise registers only events, when the photons left different output ports of the beam splitter. The other half of the events, when the photons come to the same polarization analyzer, are not resolved by this device. For simplicity, we can consider an equivalent scenario, see Fig. F2. Time-separated modes are replaced by spatialones. At each side the modes are split by two 50:50 beam splitters BS. The resolved events in the original device, cf. Fig. F1, correspond to the following events in Fig. F2, (a) clicks at the reflection port in the side $\mathrm{A}$ and the transmission port in the side $\mathrm{B}$, (b) clicks at the transmission port in the side $\mathrm{A}$ and the reflection port in the side $\mathrm{B}$. The probability of both events is $2 P_{i_{\mathrm{A}}, i_{\mathrm{B}}}\left(\theta_{\mathrm{A}}, \theta_{\mathrm{B}}\right)$, 
which should be used instead of $P_{i_{\mathrm{A}}, i_{\mathrm{B}}}\left(\theta_{\mathrm{A}}, \theta_{\mathrm{B}}\right)$ with additional $50 \%$ losses for the detection efficiency $\eta$ caused by the beam-splitters BS. However, the factor 2 disappears in Eq. (F2). Hence, the main effect of the beam-splitter BS consists in additional $50 \%$ detection losses.

The parametric down conversion source irradiates the states

$$
|\mathrm{PDC}\rangle=(\cosh \chi)^{-2} \sum_{n=0}^{+\infty} \sqrt{n+1} \tanh ^{n} \chi\left|\Phi_{n}\right\rangle
$$

where $\chi$ is the squeezing parameter, and

$$
\begin{aligned}
& \left|\Phi_{n}\right\rangle= \\
& \frac{1}{\sqrt{n+1}} \sum_{m=0}^{n}(-1)^{m}|n-m\rangle_{\mathrm{H}_{\mathrm{A}}}|m\rangle_{\mathrm{V}_{\mathrm{A}}}|m\rangle_{\mathrm{H}_{\mathrm{B}}}|n-m\rangle_{\mathrm{V}_{\mathrm{B}}} .
\end{aligned}
$$

$$
\begin{aligned}
\Phi_{\text {in }}\left(\beta_{\mathrm{H}_{\mathrm{A}}}, \beta_{\mathrm{v}_{\mathrm{A}}}, \beta_{\mathrm{H}_{\mathrm{B}}}, \beta_{\mathrm{v}_{\mathrm{B}}}\right) & =\exp \left[-\frac{\tanh ^{2} \chi\left|\beta_{\mathrm{v}_{\mathrm{A}}}\right|^{2}+\tanh ^{2} \chi\left|\beta_{\mathrm{H}_{\mathrm{B}}}\right|^{2}-\tanh \chi\left(\beta_{\mathrm{V}_{\mathrm{A}}} \beta_{\mathrm{H}_{\mathrm{B}}}+\beta_{\mathrm{v}_{\mathrm{A}}}^{*} \beta_{\mathrm{H}_{\mathrm{B}}}^{*}\right)}{1-\tanh ^{2} \chi}\right] \\
& \times \exp \left[-\frac{\tanh ^{2} \chi\left|\beta_{\mathrm{H}_{\mathrm{A}}}\right|^{2}+\tanh ^{2} \chi\left|\beta_{\mathrm{v}_{\mathrm{B}}}\right|^{2}+\tanh \chi\left(\beta_{\mathrm{H}_{\mathrm{A}}} \beta_{\mathrm{v}_{\mathrm{B}}}+\beta_{\mathrm{H}_{\mathrm{A}}}^{*} \beta_{\mathrm{v}_{\mathrm{B}}}^{*}\right)}{1-\tanh ^{2} \chi}\right] .
\end{aligned}
$$

Similar to Appendix B, we utilize Eqs. (F8)-( characteristic function at the outputs of the polarization analyzers,

$$
\begin{aligned}
\Phi_{\text {out }}\left(\beta_{T_{\mathrm{A}}}, \beta_{R_{\mathrm{A}}}, \beta_{T_{\mathrm{B}}}, \beta_{R_{\mathrm{B}}}\right) & =\int_{0}^{1} \mathrm{~d} T \mathcal{P}(T) \exp \left[-\frac{T^{2} \tanh ^{2} \chi\left(\left|\beta_{T_{\mathrm{A}}}\right|^{2}+\left|\beta_{R_{\mathrm{A}}}\right|^{2}+\left|\beta_{T_{\mathrm{B}}}\right|^{2}+\left|\beta_{R_{\mathrm{B}}}\right|^{2}\right)}{1-\tanh ^{2} \chi}\right] \\
& \times \exp \left[-\frac{T^{2} \tanh \chi\left(\beta_{T_{\mathrm{A}}} \beta_{T_{\mathrm{B}}}+\beta_{T_{\mathrm{A}}}^{*} \beta_{T_{\mathrm{B}}}^{*}+\beta_{R_{\mathrm{A}}} \beta_{R_{\mathrm{B}}}+\beta_{R_{\mathrm{A}}}^{*} \beta_{R_{\mathrm{B}}}^{*}\right) \sin \left(\theta_{\mathrm{B}}-\theta_{\mathrm{A}}\right)}{1-\tanh ^{2} \chi}\right] \\
& \times \exp \left[-\frac{T^{2} \tanh \chi\left(\beta_{T_{\mathrm{A}}} \beta_{R_{\mathrm{B}}}+\beta_{T_{\mathrm{A}}}^{*} \beta_{R_{\mathrm{B}}}^{*}-\beta_{R_{\mathrm{A}}} \beta_{T_{\mathrm{B}}}-\beta_{R_{\mathrm{A}}}^{*} \beta_{T_{\mathrm{B}}}^{*}\right) \cos \left(\theta_{\mathrm{B}}-\theta_{\mathrm{A}}\right)}{1-\tanh ^{2} \chi}\right] .
\end{aligned}
$$

In terms of the characteristic function, Eq. (F5) is given by

$$
P_{i_{\mathrm{A}}, i_{\mathrm{B}}}\left(\theta_{\mathrm{A}}, \theta_{\mathrm{B}}\right)=\int_{-\infty}^{+\infty} \mathrm{d}^{8} \beta \Phi_{\text {out }}\left(\beta_{T_{\mathrm{A}}}, \beta_{R_{\mathrm{A}}}, \beta_{T_{\mathrm{B}}}, \beta_{R_{\mathrm{B}}}\right) K_{\mathrm{C}}\left(\beta_{i_{\mathrm{A}}}\right) K_{\mathrm{C}}\left(\beta_{i_{\mathrm{B}}}\right) K_{0}\left(\beta_{j_{\mathrm{A}}}\right) K_{0}\left(\beta_{j_{\mathrm{B}}}\right),
$$

where $\mathrm{d}^{8} \beta=\mathrm{d}^{2} \beta_{T_{\mathrm{A}}} \mathrm{d}^{2} \beta_{R_{\mathrm{A}}} \mathrm{d}^{2} \beta_{T_{\mathrm{B}}} \mathrm{d}^{2} \beta_{R_{\mathrm{A}}}$,

$$
\begin{gathered}
K_{0}(\beta)=\frac{1}{\pi \eta} \exp \left[-\frac{|\beta|^{2}}{\eta}-N\right], \\
K_{\mathrm{C}}(\beta)=\delta(\beta)-K_{0}(\beta) .
\end{gathered}
$$

Substituting (F16) in (F17) we obtain an explicit form for the probabilities of simultaneous clicks at both sides,

$$
P_{\mathrm{i}_{\mathrm{A}}, \mathrm{i}_{\mathrm{B}}}\left(\theta_{\mathrm{A}}, \theta_{\mathrm{B}}\right)=\left(1-\tanh ^{2} \chi\right)^{4}\left[\left\langle\frac{\exp (-2 N)}{C_{0}+2 C_{1}+C_{\mathrm{i}_{\mathrm{A}}, \mathrm{i}_{\mathrm{B}}}}\right\rangle-\left\langle\frac{2 \exp (-3 N)}{C_{0}+C_{1}}\right\rangle+\left\langle\frac{\exp (-4 N)}{C_{0}}\right\rangle\right],
$$

where $\langle\ldots\rangle$ means averaging with respect to $T$,

$$
C_{0}=\left\{\eta^{2} T^{4} \tanh ^{2} \chi-\left[1+\left(\eta T^{2}-1\right) \tanh ^{2} \chi\right]^{2}\right\}^{2},
$$




$$
\begin{aligned}
& C_{1}=\eta T^{2}\left(1-\eta T^{2}\right)\left(1-\tanh ^{2} \chi\right) \tanh ^{2} \chi\left\{\eta^{2} T^{4} \tanh ^{2} \chi-\left[1+\left(\eta T^{2}-1\right) \tanh ^{2} \chi\right]^{2}\right\}, \\
& C_{\mathrm{T}_{\mathrm{A}}, \mathrm{T}_{\mathrm{B}}}=C_{\mathrm{R}_{\mathrm{A}}, \mathrm{R}_{\mathrm{B}}}=\eta^{2} T^{4} \tanh ^{2} \chi\left[1-\tanh ^{2} \chi\right]^{2}\left[\left(1-\eta T^{2}\right)^{2} \tanh ^{2} \chi-\sin ^{2}\left(\theta_{\mathrm{A}}-\theta_{\mathrm{B}}\right)\right], \\
& C_{\mathrm{T}_{\mathrm{A}}, \mathrm{R}_{\mathrm{B}}}=C_{\mathrm{R}_{\mathrm{A}}, \mathrm{T}_{\mathrm{B}}}=\eta^{2} T^{4} \tanh ^{2} \chi\left[1-\tanh ^{2} \chi\right]^{2}\left[\left(1-\eta T^{2}\right)^{2} \tanh ^{2} \chi-\cos ^{2}\left(\theta_{\mathrm{A}}-\theta_{\mathrm{B}}\right)\right] .
\end{aligned}
$$

Equation (F20) can now be used in Eqs. (F2)- $[$ F4), and then inserted in expression for the Bell parameter (F1). Plots for the Bell parameter as a function of the squeezing parameter $\chi$ is given in Fig. 3 .

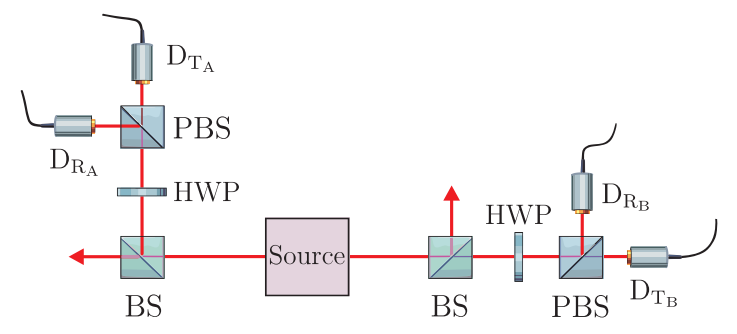

(a)

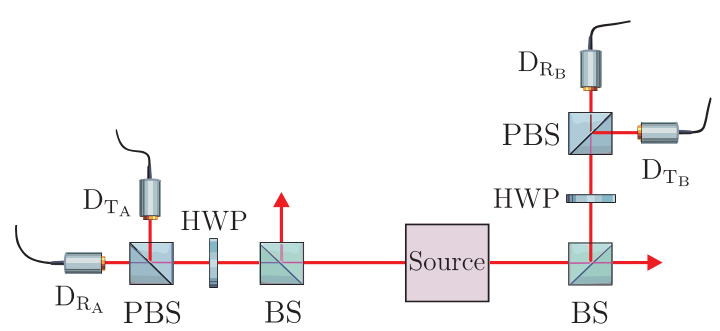

(b)

FIG. F2. Equivalent scenario for the scheme presented in Fig. F1 The time separation is replaced by the spatial separation with two beam splitters BS at each side. The schemes (a) and (b) correspond to two kinds of events, which are detected by the original device.

From the paper 7] we can extract some values of parameters. Detection efficiency is $\eta=0.25(6 \mathrm{~dB})$. Additionally we have to include the beam splitter BS losses. The total efficiency of the receiver module is $\eta=0.125$ $(9 \mathrm{~dB})$. The dark-count rate is $200 \mathrm{~s}^{-1}$ and the stray-light rate is $200 \mathrm{~s}^{-1}$. Coincidences are integrated over a $1.25 \mathrm{~ns}$ time window. This gives the mean number of stray-light and dark counts of $N=5 \times 10^{-7}$. The mean losses of the atmospheric channel are $\left\langle T^{2}\right\rangle=6.3 \times 10^{-4}(32 \mathrm{~dB})$.

\section{Appendix G: Quadrature and photon-number squeezing}

In this Appendix we consider some mathematical details related to the post-selection procedure, which improves quadrature and photon-number squeezing. We will characterize the photon-number squeezing by the
Mandel parameter,

$$
Q=\frac{\left\langle: \Delta \hat{n}^{2}:\right\rangle}{\langle\hat{n}\rangle},
$$

where $\hat{n}$ is the photon-number operator, : .. : means normal ordering. Similarly, the quadrature squeezing is characterized by the normally-ordered variance

$$
\left\langle: \Delta \hat{X}^{2}:\right\rangle
$$

of the quadrature

$$
\hat{X}=\hat{a}+\hat{a}^{\dagger},
$$

where $\hat{a}$ is the field annihilation operator. One can also use the value of squeezing in $\mathrm{dB}$ relatively to the vacuum noise,

$$
-10 \log _{10}(Q+1)
$$

for the photon-number squeezing, and

$$
-10 \log _{10}\left(\left\langle: \Delta \hat{X}^{2}:\right\rangle+1\right)
$$

for the quadrature squeezing.

Input-output relation (B8) for the normally-ordered moments can be applied for obtaining the input-output relations for squeezing factors G1 and (G2),

$$
Q_{\text {out }}=\frac{\left\langle T^{4}\right\rangle}{\left\langle T^{2}\right\rangle} Q_{\text {in }}+\frac{\left\langle\Delta \eta_{T}^{2}\right\rangle}{\left\langle T^{2}\right\rangle}\langle\hat{n}\rangle_{\text {in }},
$$

$$
\left\langle: \Delta \hat{X}^{2}:\right\rangle_{\text {out }}=\left\langle T^{2}\right\rangle\left\langle: \Delta \hat{X}^{2}:\right\rangle_{\text {in }}+\left\langle\Delta T^{2}\right\rangle\langle\hat{X}\rangle_{\text {in }}^{2},
$$

where $\left\langle\Delta T^{2}\right\rangle$ and $\left\langle\Delta \eta_{T}^{2}\right\rangle$ are the variances of the transmission coefficient $T$ and the transmission efficiency $\eta_{T}=$ $T^{2}$, respectively, cf. also Refs. 15, 20. The first terms in Eqs. G6 and (G7) resemble the standard attenuation. The second terms are non-negative and increase with increasing $\left\langle\hat{X}_{\text {in }}\right\rangle^{2}$ and $\left\langle\hat{n}_{\text {in }}\right\rangle$. They describe the effects of loss fluctuations. Hence the nonclassicality diminishes by two effects: the beam-positioning fluctuations and the brightness of the input light.

An appropriate post-selection by the transmission coefficient $T$ can improve the nonclassicality of detected 
fields. The corresponding procedure excludes the events with $T \leq T_{\min }$. The remaining events are described by the modified PDTC,

$$
\mathcal{P}_{\mathrm{ps}}(T)=\frac{1}{\overline{\mathcal{F}}} \begin{cases}\mathcal{P}(T), & \text { for } T \in\left[T_{\min }, T_{0}\right] \\ 0, & \text { else }\end{cases}
$$

where

$$
\overline{\mathcal{F}}=\overline{\mathcal{F}}\left(T_{\min }\right)
$$

is the value of exceedance $\mathrm{E} 10$ at the point $T=T_{\min }$. Post-selected PDTC (G8) can be used in Eqs. (G6) and G7 for calculation of the improved values of the corresponding squeezing factors.
In order to characterize the feasibility of the postselection procedure, we can explicitly express the squeezing factors (G6) and (G7) [and post-selected PDTC (G8)] via the exceedance $\overline{\mathcal{F}}$. For this purpose we have to resolve Eq. G9 with respect to $T_{\min }$ and substitute it in post-selected PDTC (G8). For the particular case of $d=0$ the corresponding relation,

$$
T_{\min }=T_{0} \exp \left[-\frac{1}{2}\left(-\frac{2 \sigma^{2}}{R^{2}} \ln (1-\overline{\mathcal{F}})\right)^{\frac{\lambda}{2}}\right]
$$

is obtained explicitly. 